International Journal of

Advanced Science and Convergence

\title{
Automated Driver's Assessment and Driving Simulator System
}

\author{
Rionel Belen Caldo ${ }^{1}$ and Joemar Palomaria ${ }^{2}$ \\ ${ }^{1-2}$ Multi-Axis Handlers \& Tech. Inc, Biňan, Laguna, Philippines
}

\begin{abstract}
Background/Objectives: The present invention generally relates to a driver's assessment and training simulator system designed for the improvement of the driver's screening and qualification procedures of TESDA prior to the license issuance and release in LTO. Methods/Statistical analysis: Driving Assessment and Simulation Hub (HUB) would like to propose a solution to preparing problems by designing, developing and implementing an Automated Driver's Assessment and Driving Simulator System. Findings: The present invention discloses an electronic system comprising three (3) main software components specifically, an electronic driving knowledge examination system with biometrics registration validation feature and touchscreen terminals, a driving simulation testing system with automatic scoring and up to thirty (30) heads per hour testing capacity, and a decision support system for the evaluation of the two exam results. Improvements/Applications: Driving Assessment and Simulation Hub (DASH) project team is making the Philippines a happy and safe place to drive in.
\end{abstract}

\section{Index Terms}

Driving simulator system, TESDA, LTO, Electronic system, Decision support system

\footnotetext{
Corresponding author :R. B. Caldo

rionel14_caldo@yahoo.com

- Manuscript received February 19, 2019.

- Revised March 14, 2019 ; Accepted March 22, 2019.

- Date of publication March 31, 2019.

(c) The Academic Society of Convergence Science Inc.

2619-8150 @ 2019 IJASC. Personal use is permitted, but republication/redistribution requires IJASC permission.
} 


\section{INTRODUCTION}

In the $21^{\text {st }}$ Century, there has been a significant development in mankind's way of living due to the rise of technology and globalization. This allowed society to grow and advance in many ways including its various fields in economy, education, communication, and transportation. The integration of modern and diverse technological devices and systems to the traditional processes and protocols of the government has made countless works easier, faster, and considerably more efficient.

Technology has also made its way towards the development of many aspects of man's transportation as well as the mode of acquiring the means for one. New functions, features, mechanisms, and parts are continuously being upgraded to the old and added to the new brands and models of automobiles. This attracted consumers to buy cars more than before. According to the Land Transportation Office (LTO) there are an approximately 9.3 million private car owners in the Philippines in 2017. It is equivalent to a $21.38 \%$ increase from the 7.7 million car owners in 2015 (Sarne, 2018). This does not include other motor vehicles yet such as buses, trucks, motorcycles and tricycles, trailers, and utility vehicles. One crucial issue that is being considered from this fact is also the licensing factors of the drivers of these vehicles.

The licensing of drivers in the Philippines is managed by the Land Transportation Office (LTO). It is the front line government agency that aims to provide public service for the land transportation sector. They have numerous offices and branches throughout the country to facilitate the application and renewal of both student and professional driver's licenses.

To apply and obtain a license, applicants must already have adequate prior knowledge to the many traffic rules and regulations and should also be equipped with adept skills in actual driving. Since driving the roads of the community is a very crucial thing to the safety and security of the citizens as well as valuable properties, the licensing procedure of the LTO is not and should not be taken lightly. It is the aspiring driver's responsibility to comply with all the legal and official licensing procedure. They should also pass the necessary documentary requirements to be qualified for the license.

Applicants must first obtain a certification from TESDA before or upon the license application. This is by attending the seminars conducted by TESDA that would refresh and further reinforce the applicants' knowledge of driving. The seminar also covers topics such as traffic signs and symbol identification and traffic rules and regulations. After the seminar, the applicants must then pass the written exam, which is currently in the form of the PDL
Basic Driving Theory Test, and take the practical driving test. After passing both examinations, the application shall be approved by the Approving Officer (LTO,2019).

\subsection{Background of the Study}

According to Metro Manila Accident Recording and Analysis System, an average of 299 daily vehicular accidents was recorded in Metro Manila in 2016. Richard Domingo, Metropolitan Manila Development Authority road safety unit officer, said in 2016 that $90 \%$ of road crashes were caused by driver error (MMARAS Annual Report,2016).

It was revealed that corruption and irregularities in the process of obtaining licenses from the Land Transportation Office (LTO) persist. The authorities were able to confiscate a total of 342 fake licenses in 2018. These measures contributed to the proliferation of untrained and uneducated drivers on Metro Manila's streets. The cause of this is the discrepancies happening in the actual drivers' license application. Because fixers, or the illegal third-party individuals who make arrangements for the applicants through illicit and devious ways, are rampant in every LTO offices, not all drivers who are issued with licenses actually deserve or qualified to be issued with one.

The fixers distort the actual licensing application and disrupt the assurance of upholding security, credibility, integrity, and reliability in the procedures. Unfortunately, even amidst this corrupt event happening within the licensing procedure, the LTO examiners are actually aware who among the test takers are fixers but are negligent and/or apathetic of the discrepancy.

The proponents have therefore conducted this study to help eliminate these discrepancies in the present LTO licensing procedure and improve the actual processes as well in terms of convenience, efficiency, reliability, and security. This is by integrating a Driving Simulator in the practical driving test. Upon completion and success of this project, LTO would have been able to assure that all drivers who would acquire license are only the ones that are really qualified and deserving.

\subsection{Objectives of the Study}

The general objective of this study is to develop an electronic Driving Simulation system for the improvement of the licensing procedure of the Land Transportation Office (LTO) of the Philippines. Further specific objectives are as follows:

1. To develop an online appointment reservation system as well as a biometric registration system for online and walk-in application of the driver's license. 
2. To develop web-based examination, data management, and decision support systems as a complementary components of the driving simulator.

3. To strictly ensure that all applicants to be issued with a driver's license are qualified and well-educated with the road safety and traffic rules and regulations.

4. To help prevent and eliminate "red tape" on the LTO agencies as well as help prohibit fixers and other corrupt practices.

5. To eventually and ultimately help reduce road accidents as well as destruction of lives and valuable properties.

\subsection{Significance of the Study}

The success and completion of this developmental study is significant to the general public, all the driver's license applicants, and the agencies handling it due to the following benefits:

\section{Professionalizing of the licensing process}

The actual licensing procedure will be improved in terms of speed, efficiency, convenience, credibility, security, and reliability. The period to complete the application of driver's license would be greatly reduced. The processing and its various phases would be more organized and systematic. Applicants would be able to reserve for an appointment online. The procedure of the application and processing would be automated and thus boost the security of the entire process.

\section{National certification by TESDA and LTO}

Applicants would be provided with valid certifications from the said agencies which are guaranteed added credentials to them.

3. Training provider manned by professional personnel

Those who provide the seminar and training are guaranteed professionals and are qualified to conduct such training to the applicants. Therefore the knowledge and skills shared to them are legitimately proficient and accurate.

4. The licensing procedure will be globally competitive

The actual system would be very competitive with the procedures and systems used by other advanced countries.

\section{Prevention of fake licenses}

The new procedure would prevent the spread of fake licenses since everyone has undergone valid and rigid qualification procedures. The procedure would also assure that all who gets their license are actually knowledgeable and skilled drivers.

\section{Prevention of corruption by fixers}

Since the system has improved procedure, it would help prevent corruption in the agencies and would greatly eliminate the intervention of fixers

7. The license can be recognized in other countries

Upon implementation of this system, the new licensing procedure can be emulated by other countries and thus our licenses would be globally acceptable as well.

8. The system would help promote the increase of disciplined drivers

Since everyone who gets their license is knowledgeable and deserving, our streets would have more disciplined drivers and that would improve traffic in the Philippines. No traffic, less stress, less loss of profit.

\section{Safety for everybody}

Since everyone who gets their license are not only discipline, but skilled, it would considerably lessen the number of vehicular accidents in the streets and thus would greatly lessen the number of casualties, deaths, and destruction of properties.

10. Improved traffic system and overall flow of transportation in the Philippines

Since the licensed drivers in the streets are more disciplined and there are less vehicular accidents, we would no longer be famed for a very poor traffic and transportation system.

\section{LiterATURE REVIEW}

This section deals with related literature and studies and the synthesis. The proponents gathered relevant references that will provide and help them with different thoughts, views and information in developing the study.

It was emphasized in (Wang, Zhang, Wu, \& Guo, 2017) that driving simulator is important for driving safety study. The researchers introduced three wellknown large-scale moving-base driving simulators and two fixed-base simulators. They had a research on seven main aspects namely: behavior study, driver education and training, transportation infrastructure, medicine and therapy, ergonomics, Intelligent Transportation System, and administrative method (Wang et.al, 2017).

University of Michigan Transportation Research Institute (UMTRI) offers a fixed-base simulator and Desktop Driving Simulator. They both run the latest version of Realtime Technology's (RTI) simulation engine, SimCreator (Pradhan, 2017). 
The Carnetsoft research has also its driving simulator that is programmable and fully interactive with 210 degrees surround graphics HD rendering over 6 channels. This simulator has available toolset that would enable its users to define simulation environments (Anonymous, 2017).

The research (Lynch, 2017) showcased state-ofthe-art Driving Simulator Lab in the Department of Civil, Environmental and Architectural Engineering in the University of Kansas' School of Engineering. According to the author, investigators across multiple disciplines are putting the simulator to good use, researching the behavior of the drivers, traffic engineering, signal control and new technology appearing in innovative vehicles (Lynch, 2017).

According to Driving Simulator Research (2019), many factors are influencing our driving behavior. The driving simulator allows us to examine the effects of physical, mental or visual limitations on our driving behavior in a controlled experimental environment. The authors investigated whether a (change in) road design or environment can stimulate positive driving behavior. The researchers make used of driving simulator as a research tool in the domains of road safety, behavior and road design (Driving Simulator Research, 2019).

In the research of Casutt, et.al, (2014) the results showed that the driving simulator training group has developed an improved on-road driving performance compared to the attention-training group. However, both training groups increased cognitive performance compared to the control group. This means that driving simulator training provides the potential to improve driving skills specifically in older drivers. Compared to attention training, the simulator training would become a more powerful program in an effort to increase the older drivers' safety on the road (Casutt et.al, 2014).

According to O. Shectman (2010), driving simulators show potential as a research and evaluation tool since they offer researchers with a venue in which driving behaviours can be assessed under repeatable well-controlled conditions and where data gathering is efficient and easy. However, simulation is considered an abstraction of reality in which the all-encompassing real-word is converted into a more sparse simulation. Only simpler simulation is provided which includes only specific aspects of the real world while ignoring other aspects (Shectman, 2010).

The researchers in Filtness (2013) reviewed driving simulators for driver training and assessment, which focuses on novice drivers. Accordingly, simulators enable beginners to develop and practice an array of skills without the risk of crash or the distraction of maintaining vehicle control. Presently, simulators are being used to train procedural skills and higher order cognitive skill for novice drivers. It has been analysed that after simulator training, the learners' performance have greatly improved as they begin to have superior steering control, fast progression, and fewer violations and crashes when on-road training followed. This improvement was associated with the passing of practical driving tests in simulations. Overall, research results reveal that simulators are effective at training and assessing higher order cognitive skills of novice drivers, particularly hazard perception (Filtness, 2013).

\subsection{Synthesis of Literatures}

The application of driving simulator for driver's license acquisition in the Philippines setting is promising and feasible. Similar to previous studies, the proposed can also contribute to understanding the driver's behaviour during actual driving. Several techniques and methods presented in these literatures can be acquired to further the knowledge in designing and developing the driving simulator. The use of different programming languages and graphics software are useful and can be adapted. Most of the studies conducted focused on application of driving simulator in schools and individual/personal driving behaviour assessment. However, no studies were made available to facilitate application of driving simulator to driver's license acquisition in a larger context. The novelty of this work will not only be limited to the design and development of driving simulator for LTO implementation. It will also include customized graphics design and software suits to Filipino culture. The manual method of written examination and actual examination in acquiring driver's license will be speed up by this technology. More so, another unique contribution of this work to the body of knowledge is its effective decision supporting system to decide as to whether the driver's license applicant was able to pass or fail the examination in a pre-determined period of time.

\section{Methodology}

Driving Assessment and Simulation Hub (HUB) would like to propose a solution to prevailing problems by designing, developing and implementing an Automated Driver's Assessment and Driving Simulator System which would: 1 . strictly ensure that all applicants to be issued with a driver's license are qualified and well-educated with vehicle and road safety, and traffic rules and regulations; 2. help prevent and eliminate "Red Tape" on the LTO agencies as well as help prohibit fixers and other corrupt practices, and 3. eventually 
and ultimately help reduce road accidents as well as destruction of lives and valuable properties.

In line with above stated fact analysis and target setting, the proponents exhibited the modified waterfall model for driving simulator for driver's license acquisition and management system in Fig.1. Waterfall model is a software developmental model in which the activities are being represented by blocks and are followed in sequence. The waterfall model is used in software development processes, in which the processes are seen as flowing steadily downwards (like a waterfall) (Hughey, 2009)

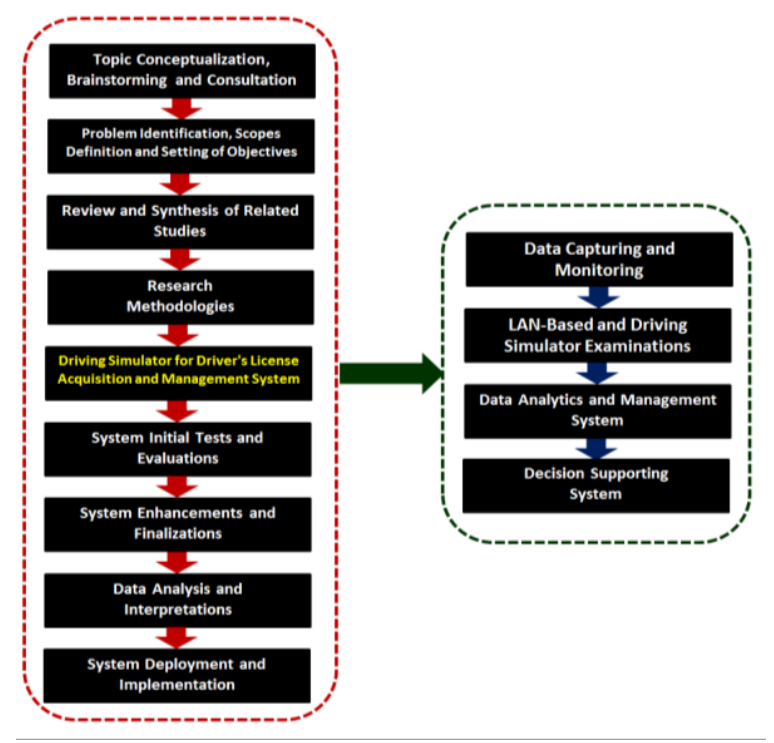

Fig. 1. Modified waterfall model for driver's license acquisition and management system

The modified waterfall model used in this study is consisted of nine blocks. As shown in the figure, the first block is the Topic Conceptualization, Brainstorming and Consultation. In this block, the proponents formulated ideas and solicit suggestions from client and asked approval from the team and stakeholders. Next phase is the Problem Identification, Scopes Definition and Setting of Objectives. In this step, the proponents were able to observe the problem in manual method of driver's license acquisition. The third important step is the Review of Related Literature and Synthesis. In this task, the proponents made comprehensive research on 10 related literatures, reviews them and summarized them. The proponents were able to identify different methods in solving the problem and were able to see the gap between existing and past studies with that of proposed. The fourth one is the Research Methodologies. In this step, the proponents were able to conceptualize the framework of the study as well as the methods that they will be using in designing and developing the system. The fifth stage is the design and development of driving simulator for driver's license acquisition and management system. This main block is further specified into four sub-sections namely: Data Capturing and Monitoring, LAN-based and Driving Simulator Examinations, Data Analytics and Management System and Decision Supporting System. These four sub-blocks are essential in providing tangible, reliable and effective driver's license acquisition and management system. The System Testing comes next, in which the proponents will need to test the system to see if there's an abnormality and if the system is producing accurate and/or reliable output. Afterwards, the system will be finalized and it will be made available to its enduser. Finally, the complete system will be deployed and implemented.

To further understand the framework of the system, the proponents constructed an InputProcess-Output (IPO) model as shown in Fig.2. In IPO perspective, the inputs are the identified hardware and the software scopes of the research. The Process block talks about the step-by-step procedures that must be followed in getting the desired output. Basically, important considerations in the process are software design and development, data capturing, data monitoring, data analysis and decision-making. The output of this system is the automatic decision (whether pass/fail) to driver's license applicants. The output will be fed back into the system (in a secured and controlled database) as it follows a closed-loop type of system in all data and information pertaining to each applicant will continuously be monitored with respect to predefined time measures.

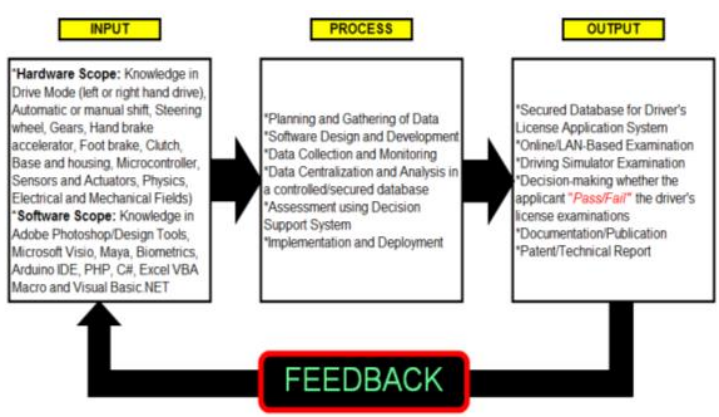

Fig. 2. IPO chart of driver's license acquisition and management system

Considering that driving simulator is a new technology which will be implemented in the Philippines, trainings must be administered prior screening and qualification of TESDA. The main goal is to prepare and educate the driver prior to the application. In order to facilitate mass training of driver's license applicants, public and private sector training sites will be established to accommodate these numbers. These training centers should obtain 
permit from screening TESDA. If they are done with the review, they can go directly to the testing center, which is administered mainly by TESDA. The applicant can apply in non-professional or professional categories. TESDA will certify and qualify the driver's license applicant. LTO will solely be responsible for issuance and release of driver's license with third party validations from TESDA and DASH.

Discussed herewith is the proposed process flow of driver's license acquisition. At first, the applicant should register his/her personal information either online or walk-in. The online registration system is applicable only for new applicant both for nonprofessional and professional applicants; he/she needs to register online for appointment based on available slot for applicant's preferred date of registration. A transaction ID will be generated by the system automatically and the applicant will bring it to nearest screening TESDA or accredited private sector with two valid IDs for verification. The person in-charge will simply have to key in the transaction ID and it should be read by the system automatically. Note: The procedure for walk-in applicant applies to online applicants during fingerprints and face validation. On the other hand, for walk-in applicants, the applicant will need to manually fill out the registration form; it will then be submitted to the designated data encoders, who will encode the information in the registration system. Transaction ID will be given to the applicant and the application will be queued for finger print registration using biometrics scanner and face capture using face recognition camera. After fingerprinting and picture taking, the applicant will now proceed with payment. Once paid, he/she can directly proceed with electronic driving examination or go to waiting rooms for some reviews. The applicant cannot proceed to driving simulator test without passing the electronic driving examination.

In the electronic driving examination, the applicant will be validated based on fingerprint. The test is consisted of 40 randomize questions for nonprofessional applicants and 60 randomize questions for professional applicants. The test questions are categorized into three (road signs and symbols, rules and regulations and situational analysis) with four selections ( $\mathrm{a}, \mathrm{b}, \mathrm{c}$ and $\mathrm{d}$ ) and it must be accomplished in one-hour. The test questions stored in the data bank are validated and provided by TESDA and
LTO. An allocated seat will be provided prior examination and the officer-in-charge can view the activities of examinees during the course of examination in his/her server screen. The examination will be administered using touchscreens by simply tapping the answer on the screen. During examination, the face recognition camera will capture the image of the examinee every five minutes. The captured image will be compared with the registered picture during recognition. If the picture did not matched, the examination will be locked automatically and it will trigger the officer-incharge that such mismatched was encountered. This will ensure that no switching of seats and/or swapping during examination were made. The results will then be printed with transaction ID as control number. The print includes the score per category and the total score and the remarks stating whether he/she can proceed to driving simulator test or should re-take the electronic driving examination. The results are classified as "Competent" or "Not Yet Competent" only. The applicant is considered passed if he got $80 \%$ and more for each category. However, if he got less than $80 \%$ even for a single category, he is considered "not yet competent" and must re-take the examination again. The duration of re-taking the examination is valid a week after up to six months only. If he failed to re-take the examination, then he will be marked "not yet competent" automatically in the system. Note that the policies and standards on taking and re-taking examinations in the discretion of TESDA and LTO will strictly be implemented. On the other hand, he can re-take the examination multiple times provided that stated rules are not violated.

After taking the electronic driving examination, the next examination is thru driving simulator. Fingerprint is needed prior to the driving simulator test for validation purposes. The driver will be scored based on a pointing system released by the LTO and modifications made by the developers on violations obtained by the driver during simulation test. Similar to electronic driving examination, face recognition cameras are also installed in each driving simulator to avoid switching and/or swapping of applicants. A report summary will be generated and it will be fed back to the main server for consolidation purposes. The driving simulator test is good for 15 minutes. There will be 10 simulators to be installed per room capable of accommodating 30 heads per hour and 
210 heads per day with approximately 45 minutes waiting time. Result of examination will be issued in a designated window with auto-generated barcode for LTO validation.

After the applicant had successful passed the two tests, he/she will be certified by TESDA to obtain driver's license from LTO. The LTO will be the ones to issue and release the driver's license to the applicant. In cases in which system goes offline, hard copy can be used by the applicant instead. The applicant can go to LTO to obtain license with necessary paper documentation. LTO can call for validations, if okay then they can proceed with driver's license issuance. To strengthen foolproofing validations and quality control, three institutions will take charge of database monitoring and linkage: 1) LTO, 2) TESDA and 3) DASH.

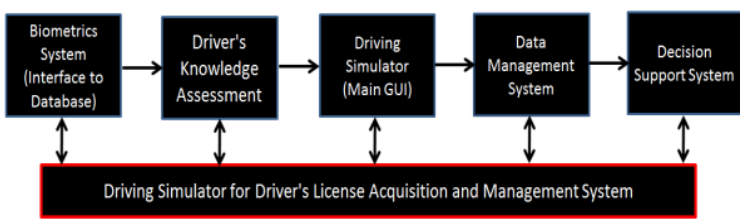

Fig. 3. Block diagram of the system

Fig. 3 shows the data flow of the whole system. The diagram is composed of five main processes. The interface of the system to centralized database will be facilitated using Biometrics. The LAN-Based Examination and the Driver Simulator (Main GUI) will require biometrics from the user to fetch information from the database and to transmit back to the main database the results of examination. The information will be managed and analysed using algorithm to be designed by the proponents and a decision will be made based on this analysis as to whether the driver's license applicant was able to pass/fail the examination and if he/she is entitle to receive driver's license. The data uploaded in the database is done through Visual Basic.Net and PHP.

To further understand the entire flow of the system, the proponents provided the block diagrams of each system in Figures 4, 5 and 6 for LAN-based examination, driving simulator examination and decision support system for driver's license acquisition respectively. Each step and process of each system was detailed and simplified.

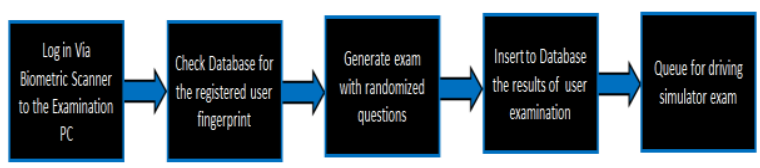

Fig. 4. Block diagram of the LAN-based examination

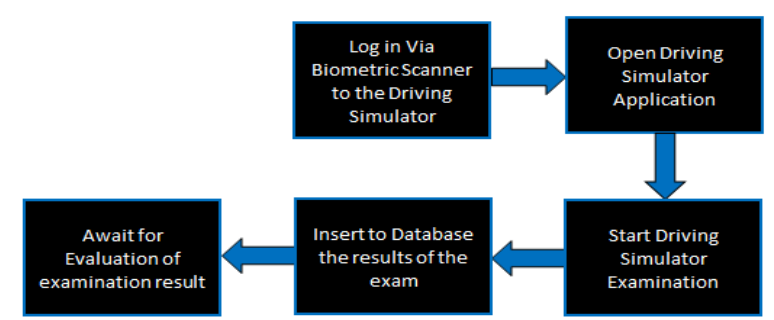

Fig. 5. Block diagram of the Driving Simulator examination

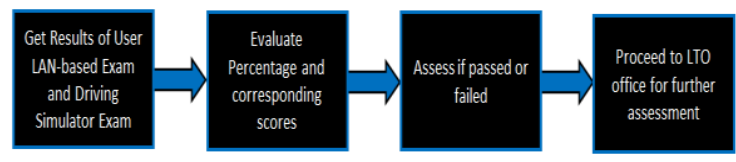

Fig. 6. Block diagram of the Decision Support System for driver's license acquisition

The flowchart of the whole system is as detailed in Figure 7. The flowchart presents the entire flow of the system from registration which could be done either online or walk-in, driver's knowledge assessment examination and driving simulator test up to acquisition and issuance of driver's license.

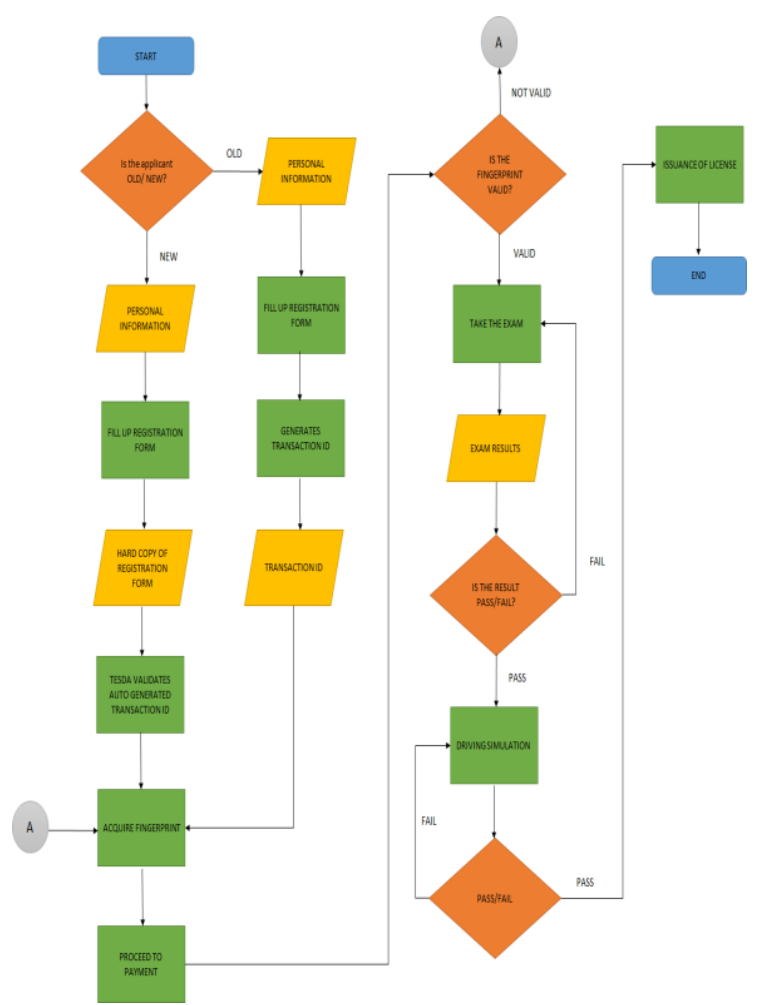

Fig. 7. Flowchart of the whole system

\section{SUMMARY OF THE INVENTION}

The system is composed of three (3) major programs. First is the Electronic Driving Examination System that runs on a personal 
computer (PC) with a touchscreen terminal. This is where the applicants will login via biometrics scanner to validate registration. The system then checks the database for registered user's fingerprint and generates an exam with randomized questions. The applicant's knowledge concerning traffic signs and symbols, traffic rules and regulations, and environment very much similar to the real-life roads and traffic situational analysis will be assessed. After the exam, the applicant will then be queued for driving simulation test.

The applicant will login again via biometric scanner in the $2^{\text {nd }}$ major program of the system, the Driving Simulator. The simulator application opens to assess the driving skills of the applicant. The driving simulator is a $3 \mathrm{D}$ simulation system wherein the applicants' actual driving skills are tested in a situational. The applicants' abilities to accurately and effectively respond to different situations and various rules and regulations are assessed as well. Both the electronic examination and driving simulator systems are developed using C\#, Oracle language and under the .Net framework. The 3D graphical animations of the simulation environment, on the other hand, is created using the Unity application and runs on a terminal installed in a customized driver's seat enclosure to imitate real-life driver-vehicle setup.

Finally, the system is also composed of the Decision Support System wherein the results from the electronic exam and driving simulator systems are evaluated and computed. This system is responsible for deciding whether the applicant has passed and is qualified for license issuance and release in accordance to the scores of the two exams.

Below are some screenshot images of the GUI of DASH's Registration System for driver's license applicants:

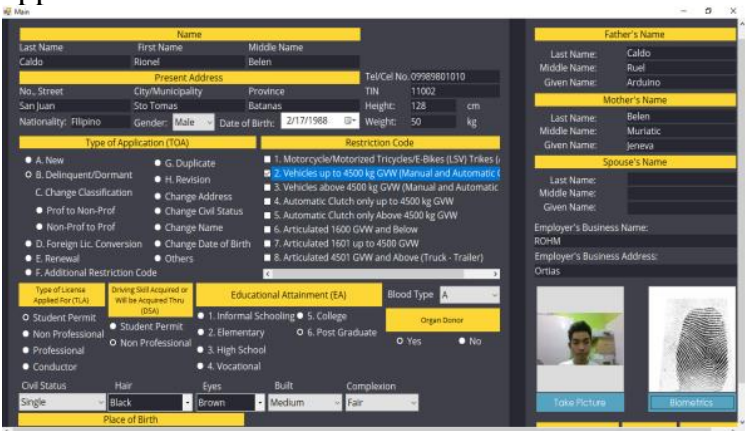

Fig. 8. Screenshot of the Graphics User Interface (GUI) of the Manual Registration System
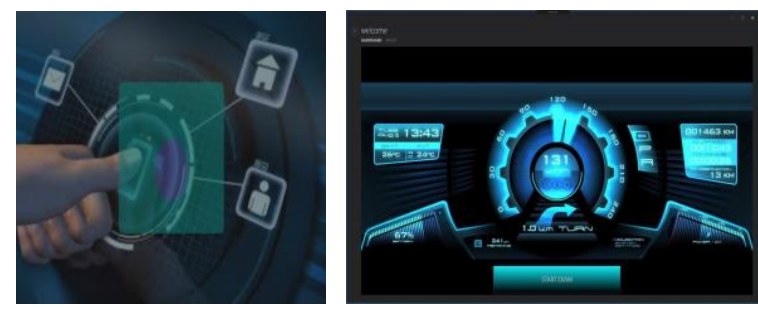

Fig. 9. Screenshot of the Graphical User Interface (GUI) of the Electronic Driving Examination System as examinees start the exam upon logon

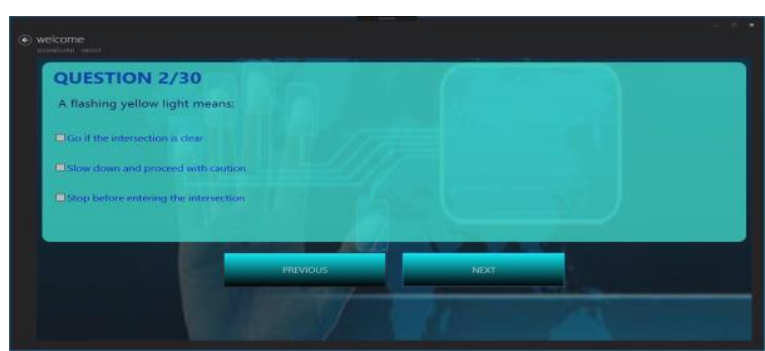

Fig. 10. Screenshot of a sample question in the Electronic Driving Examination System

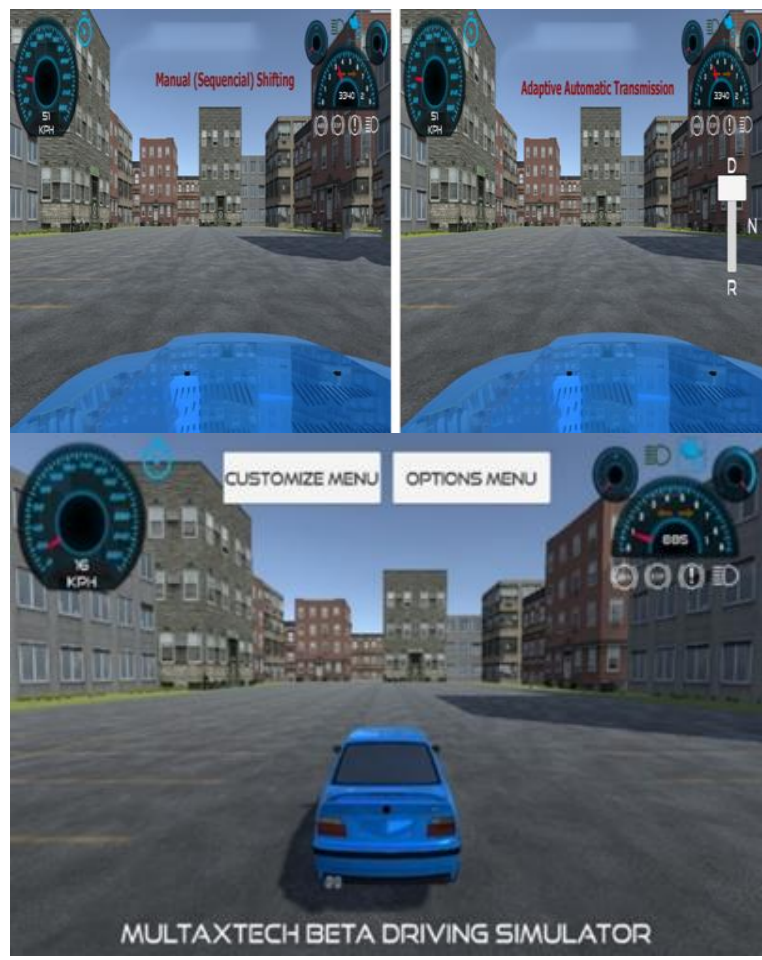

Fig. 11. Screenshot of Driving Simulator Testing System

\section{CONCluSion AND FUture DIRECTIVES}

In this work, the proponents presented an innovation on automated driver's assessment and driving simulator system which was already presented to TESDA last April 21, 2018 and endorsed to DOTr for implementation in the Philippines. As presented in this paper, it would be beneficial implementing in the Philippines for the following compelling reasons: 1) Since everyone who gets a Driving NC2 Certificate is worthy, knowledgeable and competent, then our streets would have more disciplined driver, 2) This would result to less traffic, less stress and increase in 
productivity, 3)The system shall considerably minimized road crashes resulting to saving limbs, lives and properties and 4) The actual system would be very competitive with the procedures and systems used by other advanced countries.

In a nutshell, this project would be:

- Beneficial for the whole country

- Eliminates corruption

- Ensures drivers' competency

- Ensures safe and happy environment

Below are the claims of the invention as stipulated in its specifications document applied for patent:

1. A system comprising: an Electronic driving knowledge assessment and examination system; a driving simulation system; and a decision support system.

2. The invention of claim 1 wherein said electronic driving knowledge assessment and examination system is installed in a touchscreen terminal with a fingerprint biometrics registration validation; a randomized question generation; and a driving simulation exam queuing feature.

3. The invention of claim 1 wherein said driving simulation system is installed in a customized driver's seat enclosure with a biometric login and a 3D graphical traffic environment animation.

4. The invention of claim 1 wherein said decision support system connects to the said electronic knowledge assessment and driving simulation testing systems for exam results evaluation.

Driving Assessment and Simulation Hub (DASH) project team is making the Philippines a happy and safe place to drive in.

\section{ACKNOWLEDGEMENT}

The authors acknowledge Jesus Christ, who is the ultimate source of knowledge and wisdom. They would like to thank AllTESDAPhil in collaboration with Driving Assessment and Simulation Hub (DASH) for this research endeavor. The proponents are grateful as well to Technical Education and Skills Development Authority (TESDA) and Land Transportation Office (LTO) for their favorable support in granting the project, its dissemination and publication.

\section{REFERENCES}

[1] Anonymous. (2017). Research Driving Simulator. [Online]. Available: http://cs-drivingsimulator.com/research-driving-simulator/.

[2] Casutt, G. et al. (2014). The drive-wise project: driving simulator training increases real driving performance in healthy older drivers. [Online].
Available: https://www.ncbi.nlm.nih. gov/pmc/ articles/PMC 4026721/

[3] Driving Simulator Research. (2019). [Online]. Available: https://www.uhasselt.be/UH/IMOBEN/IMOB_EN-Research/Driving-simulatorresearch.html.

[4] Filtness, A. (2013). Simulators for skill acquisition training and assessment, and their impact on road safety. [Online]. Available: https://www.google.com.ph/url?sa=t\&rct=j\&q=\&esrc $=$ s\&source=web\&cd=27\&ved=0ahUKEwj836_orJD XAhVCv7wKHapwDA84FBAWCEEwBg\&url=https $\% 3 \mathrm{~A} \% 2 \mathrm{~F} \% 2 \mathrm{Fwww}$.tmr.qld.gov.au\%2F$\% 2$ Fmedia $\% 2$ FSafety $\% 2$ Froadsafety $\% 2$ FRoadsafety-research-reports $\% 2$ Freport-2simulators.pdf\%3Fla\%3Den\&usg=AOvVaw1Vsk51M tijS73n4VQUFHWc.

[5] Hughey. D. (2009). Comparing traditional systems analysis and design with agile methodologies. [Online]. Available: http://www.umsl.edu/ hugheyd/ is6840/waterfall.html.

[6] LTO License and Permit Issuance. (2019). [Online]. Available: $\quad$ http://www.lto.gov.ph/lto-forms.html \#slider.

[7] Lynch, B. (2017). New Driving-Simulator Lab accelerates Research into Driver Behavior and Vehicle Technology, June 8, 2017.

[8] MMARAS Annual Report. (2016). [Online]. Available:https://www.foi.gov.ph/requests/aglzfmVm b2ktcGhyHgsSB0NvbnRlbnQiEU1NREEtMTMzNz Y5OTcwNjMxDA

[9] Pradhan, A. (2017). UMTRI's Driving Simulator. [Online]. Available: http://www.umtri.umich.edu/ what-we-offer/driving-simulator.

[10] Sarne, V. (2017) How many new cars were added to our roads?. [Online]. Available: https://visor.ph/ wisdom/how-many-new-cars-were-added-to-ourroads-in-2017/

[11] Shectman. (2010). Validation of Driving Simulators. [Online]. Available: http://host.uniroma3.it/riviste/ats/ special\%20issue\%202010/paper\%205_53-62_A.pdf.

[12] Wang, Y., W. Zhang, S. Wu \& Y. Guo. (2017). Simulators for Driving Safety Study - A Literature Review, Lecture Notes in Computer Science, 4563, 584-593. 